\title{
The proof of the pudding is in the eating: an outbreak of emetic syndrome after a kindergarten excursion, Berlin, Germany, December 2007
}

G O Kamga Wambo ${ }^{1,2}$, F Burckhardt' ${ }^{1,2}$, C Frank $^{1}$, P Hiller ${ }^{3}$, H Wichmann-Schauer ${ }^{3}$, I Zuschneid ${ }^{4}$, J Hentschke , T Hitzbleck $^{6}$, M

Contzen ${ }^{7}$ M Suckau ${ }^{8}, K$ Stark (starkk@rki.de) ${ }^{1}$

1. Department for Infectious Disease Epidemiology, Robert Koch Institute, Berlin, Germany

2. Postgraduate Training for Applied Epidemiology (PAE, German Field Epidemiology Training Programme), Robert Koch Institute, Department for Infectious Disease Epidemiology, Berlin, Germany

3. Federal Institute for Risk Assessment, Berlin, Germany

4. Public Health Authority Friedrichshain-Kreuzberg, Berlin, Germany

5. Institute for Food Safety, Drugs and Animal Health, Berlin, Germany

6. Veterinarian and Food Safety Authority, Tempelhof-Schöneberg, Berlin, Germany

7. Chemical and Veterinary State Laboratory (Chemisches und Veterinäruntersuchungsamt, CVUA), Stuttgart, Germany

8. Senate for Health, Environment and Consumer Protection, Berlin, Germany

Kamga Wambo GO, Burckhardt F, Frank C, Hiller P, Wichmann-Schauer H, Zuschneid I, Hentschke J, Hitzbleck T, Contzen M, Suckau M, Stark K. The proof of the pudding is in the eating: an outbreak of emetic syndrome after a kindergarten excursion, Berlin, Germany, December 2007. Euro Surveill. 2011;16(15):pii=19839.

Available online: http://www.eurosurveillance.org/ViewArticle.aspx?Articleld=19839

An outbreak of food poisoning (emetic syndrome) occurred in three kindergartens (A, B and C) in Berlin, Germany, on 3 December 2007 after an excursion during which food was served. We conducted a retrospective cohort study among the kindergarten children and personnel who participated in the trip. The overall attack rate among the 155 participants was $30 \%$. It was $31 \%$ among the 137 children (aged two to six years) and $17 \%$ among adults $(n=18)$. The consumption of rice pudding was significantly associated with disease. Among those who ate rice pudding, the attack rate was $36 \%$, compared with $0 \%$ for non-eaters (relative risk: infinite, p $<0.001$, aetiological fraction: $100 \%$ ), but differed significantly between kindergartens A ( $43 \%)$, B $(61 \%)$ and C (3\%), probably because groups were served from different pots. Bacillus cereus sensu stricto was identified from one vomit sample. The clinical and epidemiological characteristics suggest that $B$. cereus emetic toxin (cereulide) was the causative agent, although it could not be proven in the single vomit isolate. Inadequate food handling most probably led to the outbreak. Single-portion ready-to-eat rice pudding was recommended for subsequent excursions and no further cases of food poisoning occurred.

\section{Introduction}

In some outbreaks of infectious gastroenteritis, emesis predominates. The emetic syndrome is characterised by acute-onset nausea and vomiting. The most common pathogens associated with emetic syndrome are enterotoxin-producing Staphylococcus aureus and emetictoxin-producing Bacillus cereus [1-5]. Staphylococcal food poisoning results from the ingestion of enterotoxins preformed in food by enterotoxigenic strains of coagulase-positive staphylococci, mainly $S$. aureus. Several staphylococcal enterotoxins are heat-stable.
The range of the incubation period is 0.5 to 8 hours. $B$. cereus is a spore-forming microorganism, which can cause both emetic and diarrhoeal types of disease. It occurs ubiquitously in the environment (e.g. in soil) and may also be found in various foodstuffs. The emetic type of disease is caused by a heat-stable peptide toxin (cereulide): the incubation period ranges from 0.5 to 6 hours. The illness usually does not persist longer than 24 hours but severe and fatal outcomes have been reported $[6,7]$. The toxin is produced in food when the organism multiplies at ambient temperature for several hours (e.g. if the food is inadequately stored after cooking) [5]. Emetic outbreaks due to $B$. cereus have mainly been linked to starchy foods such as rice, pasta and pastry [2].

Norovirus is also a common cause of outbreaks of acute gastroenteritis, with emesis as a prominent symptom. Infection can arise from contact with or airborne transmission from fomites, as well as faecal-oral and foodborne transmission.

Although outbreaks of acute gastroenteritis are notifiable in most countries, the number of toxin-related food poisoning outbreaks is largely underestimated because the disease is often mild and self-limiting, and laboratory detection (toxin testing) is not routinely performed.

On 3 December 2007, a kindergarten (A) reported cases of emesis among children and its personnel to the local health authority. In the morning of the same day they had been on an excursion on a local tram that included catering on the platform at the tram's final destination. Preliminary investigations by the local health authority confirmed the outbreak in this and 
two other participating kindergartens (B and C) from another Berlin district.

We conducted an investigation immediately after the outbreak had come to our attention, to assess its scope, to identify the causative agent, and to determine the risk factors and the vehicle of infection in order to prevent further outbreaks.

\section{Methods}

\section{Case finding}

The tram excursion took place on the morning of 3 December 2007 between 09:00 and 10:00. On the following day, cases among the kindergarten groups were identified by the local health authorities. On 6 December, we obtained the addresses and telephone numbers of the kindergartens from the local health authorities. Food safety authorities provided the address of the caterer and the list of food items served during the excursion.

Exploratory interviews at the kindergartens were conducted on 7 December and showed that the staff who had accompanied the excursion clearly remembered the relevant epidemiological details (e.g., disease status and food consumption) of the children. Therefore we interviewed the kindergarten personnel using a standardised questionnaire on the children's and their own clinical symptoms, time of disease onset, type and duration of symptoms, secondary spread among family members, food consumption and demographic data.

\section{Case definition}

We defined a case as a person who attended the excursion on 3 December 2007 between 09:00 and 10:00 and presented with vomiting, abdominal pain or diarrhoea within 24 hours after the excursion.

\section{Cohort study}

We conducted a retrospective cohort study among children and personnel of the three affected kindergartens. We described cases by date and time of disease onset. Age group-specific and kindergarten-specific attack rates were calculated. We also calculated food-specific attack rates, aetiological fractions, relative risks and $95 \%$ confidence intervals. Data were also stratified by kindergarten to compare the results between the kindergartens. We used EpiData for data entry and SPSS software, version 15.0, for statistical analysis.

\section{Laboratory methods \\ Human samples}

Stool samples $(n=10)$ and one available vomit sample were tested (at the Institute for Food Safety, Drugs and Animal Health) for various enteric pathogens (Salmonella, Campylobacter, Escherichia coli and other enterobacteria, Yersinia enterocolitica, S. aureus, $B$. cereus and viruses such as norovirus, adenovirus, rotavirus and astrovirus). For detection of bacteria, routine culture methods were used, and for viruses, PCR and antigen tests were carried out. In the routine laboratory investigations of the stool and vomit samples, no tests for staphylococcal enterotoxins or $B$. cereus emetic toxin were performed.

An isolate of presumptive $B$. cereus from the vomit sample was tested for $B$. cereus cereulide production using liquid chromatography-tandem mass spectrometry (LC-MS/MS), and for the presence of the cereulide synthetase (ces) gene using PCR. For species differentiation, we used Fourier transform infrared spectroscopy [8].

For LC-MS/MS analysis of cereulide, bacteria were directly extracted with methanol during ultrasonification [8]. Chromatographic separation took place on a C8 column with a buffer/methanol gradient, a triple quadrupole mass spectrometer with positive electrospray ionisation run in multiple reaction monitoring mode (mass-to-charge ratio 1,170.7 $\left[\mathrm{M}+\mathrm{NH}_{4}\right]+\cdots$... $940.2 ; 1,170.7 \cdots \cdots$; $741.4 ; 1,170.7 \cdots$; $499.2 ; 1,170.7 \cdots$ 357.2) was used for detection.

PCR to detect the $B$. cereus ces gene was performed using primers ces_TaqM_for and ces_TaqM_rev with probe ces_TaqM_probe (TIB-MolBiol, Berlin, Germany),

\section{TABLE 1}

Cohort characteristics with attack rates, outbreak of emetic syndrome following kindergarten excursion, Berlin, Germany, December 2007

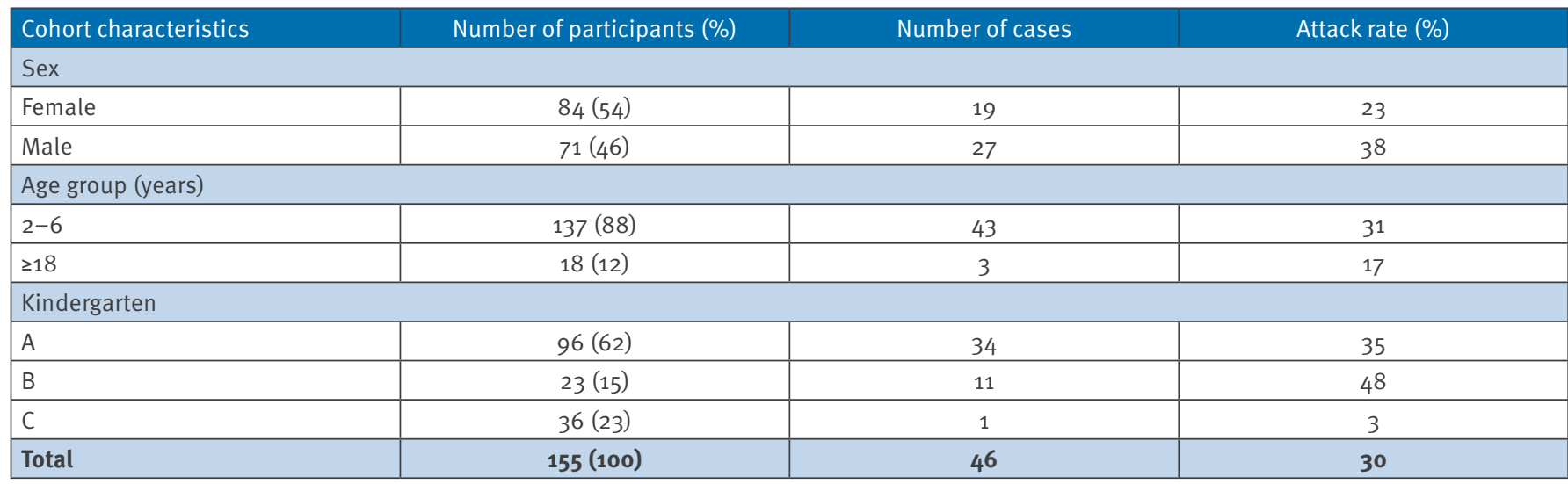


as described by Fricker et al. [9]. For more details, see Rau et al. [8].

\section{Food leftovers}

Two unopened tetrapaks of the rice pudding that had been used, and retain samples (obtained from the caterer) of spray cream, cinnamon-sugar mix, gingerbread and two opened bags of cocoa powder were tested for Salmonella, staphylococci, B. cereus, Campylobacter, E. coli, Listeria monocytogenes, Clostridium perfringens, Enterobacteriaceae, Pseudomonas and norovirus. Leftovers of heated rice pudding eaten on the tram platform were not available for testing.

\section{Environmental investigation}

Local health and food safety authorities inspected the caterer's facilities used on the tram platform and the cleaning facilities in the caterer's office. The caterer was interviewed regarding food purchase, transport and storage, the facilities on the tram platform during the excursion (stand, water and electricity supply), the preparation process of food items and drinks served during the excursions, and on the cleaning procedures of the cookware.

\section{Results}

\section{Descriptive epidemiology}

Overall, 155 persons (137 children, 17 kindergarten staff and one of the children's mother) from the three kindergartens participated in the excursion. The cohort characteristics are shown in Table 1. In total, 46 participants ( 43 children aged two to six years, and three adults) met the case definition (attack rate: $30 \%$ ). The attack rate was higher among the children than among the adults, and differed significantly by kindergarten (p<0.001).

\section{FIGURE}

Cases by hour of symptom onset, outbreak of emetic syndrome following kindergarten excursion, Berlin, Germany, 3 December 2007 ( $n=35)$

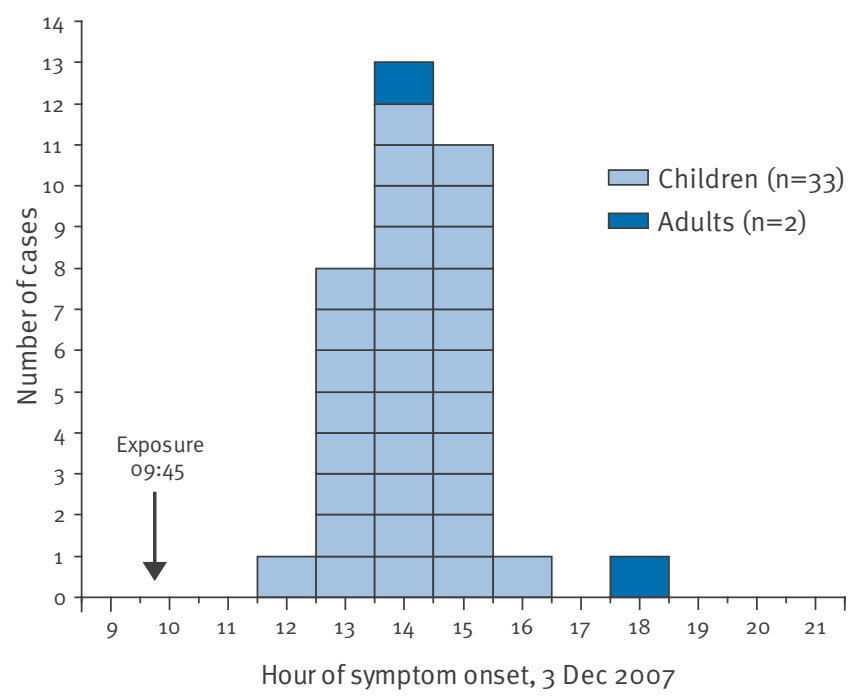

The predominant symptoms were vomiting $(n=39)$, and abdominal pain $(n=29)$. Diarrhoea was reported only in one person. Nobody was hospitalised and all cases recovered within one day. There were no secondary cases among household members.

The food items served during the excursion (at 09:45) were ready-to-eat rice pudding (from one-litre tetrapacks) that was heated before serving (served with cinnamon-sugar mix), cocoa drinks with and without whipped cream, and gingerbread. According to the personnel in Kindergarten $\mathrm{C}$, the only person who became ill in this kindergarten recalled having been served from a different pot than that used for the other participants from this kindergarten. The participants had no other common meals before or after the excursion.

In all cases, symptoms started within a few hours after the end of the excursion. Detailed information about the time (hour) of symptom onset was available for 35 cases. Onset of symptoms began in the first case on 3 December, 2.5 hours after the meal had been served (Figure). The median time between the meal and symptom onset (the median incubation period) was four hours. No cases had onset of symptoms later than eight hours after the meal.

\section{Cohort study}

Of the food items served during the excursion, only consumption of rice pudding was significantly associated with illness in the cohort study. The relative risk was infinite (Table 2) with p<0.001, and all cases could be explained by the consumption of rice pudding (aetiological fraction: $100 \%$ ). After stratifying by kindergarten (Table 3), the consumption of rice pudding remained associated with disease.

\section{Laboratory results}

One vomit sample was provided on the day of symptom onset (3 December 2007); 10 stool samples were provided after 6 December. 'Presumptive $B$. cereus' (collective name for $B$. cereus sensu strictu, $B$. thuringiensis and closely related bacilli), isolated from the culture of the vomit sample, was analysed by LC-MS/MS for cereulide production and for the presence of the ces gene by PCR: both analyses gave negative results [8]. No cereulide could be detected in the vomit sample itself. The isolate, initially described as presumptive $B$. cereus, was identified as $B$. cereus sensu stricto (non-cereulide producing) by Fourier transform infrared spectroscopy.

All stool samples taken within a few days after symptom onset and all food samples were negative for all tested pathogens.

\section{Environmental results}

All food items had been purchased by the caterer at the end of November 2007 and had been stored in the boot of the caterer's car until 1 December 2007. One similar excursion had taken place on 1 December 2007, with 
the same tram and the same catering company, but no outbreak occurred.

On both excursions, pots in an electric water-bath were used to heat the rice pudding and to keep it warm on the tram platform. The caterer stated that after the meal of the first excursion (on 1 December), rice pudding remnants had been scraped out of the pots and the pots were cleaned superficially in a wash-hand basin in an improvised kitchen in the caterer's office. According to the caterer, no food leftovers were served on 3 December. On that day, since there were more participants than in the previous excursion, the caterer used three additional cooking pots to heat up the rice pudding. The electricity supply was temporarily interrupted (due to a blown fuse) during the food preparation on 3 December.

\section{Discussion}

There is strong epidemiological evidence that the vehicle of the outbreak was rice pudding served during the excursion on 3 December 2007: the narrow epidemic curve indicated a common source of infection. All cases of emetic syndrome could be explained by the consumption of rice pudding from some of the pots used, while other food items were not associated with illness. Unfortunately, only one vomit sample was available for testing: no leftovers of the rice pudding portions served were available. This substantially hampered the laboratory investigations and no causative agent could be unambiguously identified. However, the clinical characteristics of this outbreak - including the short incubation period (of only a few hours), vomiting as the main symptom and the short self-limiting course of the disease - are typical for $B$. cereus emetic toxins or $S$. aureus enterotoxin. The fact that rice pudding was the likely vehicle suggests that this outbreak was caused by $B$. cereus cereulide. Starchy food products, including rice dishes, have been described as typical vehicles in $B$. cereus toxin outbreaks $[2,5,10]$. However, $S$. aureus cannot be ruled out as the responsible pathogen. In both scenarios of $B$. cereus or S. aureus having caused the outbreak, the food contamination must have occurred at least several hours before serving because this minimum time is required for pathogen multiplication or germination (in case of $B$. cereus) and for toxin production $[2,5]$. It is very unlikely that the unopened commercial readyto-eat tetrapacks were contaminated: had they been, more outbreaks would have been expected, given the wide distribution of these products. Since the cinnamon-sugar mix was added to the rice pudding only

\section{TABLE 2}

Food-specific attack rates, aetiological fraction and relative risks with 95\% confidence intervals, outbreak of emetic syndrome following kindergarten excursion, Berlin, Germany, December 2007

\begin{tabular}{|c|c|c|c|c|c|c|}
\hline $\begin{array}{l}\text { Food items } \\
\text { served during } \\
\text { the excursion }\end{array}$ & $\begin{array}{l}\text { Eaten } \\
\text { by the person }\end{array}$ & $\begin{array}{l}\text { Number of participants } \\
\text { who developed symptoms } \\
\text { and with available information } \\
\text { about food items consumed } \\
n=46\end{array}$ & $\begin{array}{l}\text { Overall number of participants } \\
\text { with available information } \\
\text { about food items consumed }\end{array}$ & $\begin{array}{c}\text { Attack rate } \\
(\%)\end{array}$ & $\begin{array}{l}\text { Aetiological } \\
\text { fraction } \\
(\%)\end{array}$ & $\begin{array}{c}\text { Relative risk } \\
(95 \% \mathrm{Cl})\end{array}$ \\
\hline \multirow{2}{*}{ Rice pudding } & Yes & 46 & 129 & 36 & 100 & $\infty$ \\
\hline & No & 0 & 24 & 0 & - & - \\
\hline \multirow{2}{*}{ Cocoa drink } & Yes & 37 & 124 & 30 & 79 & $1.0(0.5-1.9)$ \\
\hline & No & 7 & 23 & 30 & - & - \\
\hline \multirow{2}{*}{ Whipped cream } & Yes & 5 & 22 & 23 & 11 & $0.8(0.3-1.8)$ \\
\hline & No & 32 & 109 & 29 & - & - \\
\hline \multirow{2}{*}{ Gingerbread } & Yes & 2 & 22 & 9 & 4 & $0.3(0.1-1.1)$ \\
\hline & No & 34 & 105 & 32 & - & - \\
\hline
\end{tabular}

a For rice pudding: $n=153$; cocoa drink: $n=147$; whipped cream: $n=131$; gingerbread: $n=127$.

\section{TABLE 3}

Stratified analysis by kindergarten for rice pudding-specific attack rates, aetiological fraction and relative risks, outbreak of emetic syndrome following kindergarten excursion, Berlin, Germany, December 2007

\begin{tabular}{|c|c|c|c|c|c|c|}
\hline Kindergarten & $\begin{array}{l}\text { Rice pudding } \\
\text { eaten by the } \\
\text { person }\end{array}$ & $\begin{array}{c}\text { Number of cases } \\
\text { who consumed } \\
\text { rice pudding and developed } \\
\text { symptoms } \\
n=46\end{array}$ & $\begin{array}{c}\text { Overall number of participants } \\
\text { with available information } \\
\text { about rice pudding } \\
\text { consumption } \\
n=153\end{array}$ & $\begin{array}{l}\text { Attack rate } \\
(\%)\end{array}$ & $\begin{array}{c}\text { Aetiological fraction } \\
(\%)\end{array}$ & $\begin{array}{l}\text { Relative } \\
\text { risk }\end{array}$ \\
\hline \multirow{2}{*}{ A } & Yes & 34 & 79 & 43 & 100 & $\infty$ \\
\hline & No & 0 & 16 & 0 & - & - \\
\hline \multirow{2}{*}{ B } & Yes & 11 & 18 & 61 & 100 & $\infty$ \\
\hline & No & 0 & 4 & 0 & - & - \\
\hline \multirow{2}{*}{ C } & Yes & 1 & 32 & 3 & 100 & $\infty$ \\
\hline & No & 0 & 4 & 0 & - & - \\
\hline
\end{tabular}


shortly before consumption it can be ruled out as the vehicle of the outbreak.

Unfortunately, in the initial microbiological investigations the human and food samples had not been tested specifically for the presence of $B$. cereus toxins and $S$. aureus enterotoxins. In such outbreaks, human and food samples should be obtained and tested in a timely manner, not only for the usual pathogens (bacteria and viruses) but also for the relevant toxins, using the appropriate tests. The $B$. cereus-like strain isolated from the only vomit sample tested negative for cereulide or the ces gene. However, it is conceivable that emetic-toxin-producing $B$. cereus strains as well as non-toxin-producing strains were present in the rice pudding, but could not be detected in the vomit sample. The presence of $B$. cereus in the vomit sample and the absence of this agent from the unopened package of rice pudding is consistent with a scenario of $B$. cereus spores (including toxin-producing and nontoxin-producing strains) having contaminated the rice pudding after the tetrapacks were opened. The spores may have germinated and multiplied in remnants of the rice pudding left in the pots during an inadequate cleaning and storage process between the first and second excursion. This scenario is supported by the fact that not all of the pots appear to have contained contaminated rice pudding.

The fact that children from three kindergartens participated in the excursion and were affected by emetic syndrome shortly afterwards (although with attack rates differing by kindergarten) clearly pointed to a common source related to the excursion. This epidemiological pattern narrowed the spectrum of causative agents to toxin-producing agents. This shift of focus when patients from more than one setting are affected is an important epidemiological practice that is not always appreciated. If only one kindergarten had been involved, the investigation would have needed to also examine potential earlier sources of exposure to other pathogens such as norovirus and rotavirus. In this outbreak, the epidemiological investigation started shortly after the outbreak had been detected and the kindergarten staff clearly remembered the few food items consumed by the children. However, in other outbreak investigations, if substantial time elapses between symptom onset and epidemiological data collection (e.g. standardised interviews) or if many different food items had been served recall bias may be a major problem.

Although the environmental investigations did not determine the source of the food contamination, it revealed several breaches in food hygiene regarding cleaning of the cooking pots between the first and second excursion, as well as incorrect holding times and temperatures of food.

The epidemiological findings in this outbreak are consistent with other published $B$. cereus-associated food-borne outbreaks $[11,12]$. It should also be noted that food can be contaminated at the same time by different strains of presumptive $B$. cereus $(B$. cereus sensu stricto, $B$. thuringiensis, $B$. weihenstephanensis), which can be difficult to discern in some cases of food poisoning [13-15]. Also contamination with mixed cultures of emetic and non-emetic $B$. cereus sensu stricto can occur that can only be revealed by the testing of several isolates [8]. Detection of the $B$. cereus toxin as well as $S$. aureus enterotoxin in human and food samples is not straightforward and may require advanced methods in specialised laboratories [13-15].

Mobile caterers and persons responsible for such excursions should be aware of the potential risk of outbreaks caused by bacterial toxins. In order to prevent $B$. cereus spores from germinating and producing heatstable cereulide, caterers need to ensure that food leftovers are discarded or refrigerated at a temperature below $10^{\circ} \mathrm{C}$ and, if stored, that they are reheated thoroughly (at least $65^{\circ} \mathrm{C}$ ) before consumption.

In presumed food-poisoning outbreaks, stool and vomit samples from a substantial number of patients as well as relevant food leftovers and their ingredients should be obtained and investigated, not only for pathogens but also for the relevant toxins by appropriate tests. If $B$. cereus is identified, it is useful to further analyse several isolates from the culture to identify toxin-producing $B$. cereus strains.

In the light of our study, we recommended using single-portion, ready-to-eat rice pudding packs during future kindergarten field trips. No further food-borne outbreaks related to such excursions were reported to the local health authorities.

\section{References}

1. Balaban N, Rasooly A. Staphylococcal enterotoxins. Int J Food Microbiol. 2000;61(1):1-10.

2. Kramer JM, Gilbert RJ. Bacillus cereus and other Bacillus species. In: Doyle MP, editor. Foodborne bacterial pathogens. New York: Marcel Dekker; 1989. p. 21-70.

3. Ostyn A, De Buyser ML, Guillier F, Groult J, Felix B, Salah $S$, et al. First evidence of a food poisoning outbreak due to staphylococcal enterotoxin type E, France, 2009. Euro Surveill. 2010;15(13):pii=19528. Available from: http://www. eurosurveillance.org/ViewArticle.aspx?Articleld=19528

4. Schmid D, Fretz R, Winter P, Mann M, Höger G, Stöger A, et al. Outbreak of staphylococcal food intoxication after consumption of pasteurized milk products, June 2007, Austria. Wien Klin Wochenschr. 2009;121(3-4):125-131.

5. Stenfors Arnesen LP, Fagerlund A, Granum PE. From soil to gut: Bacillus cereus and its food poisoning toxins. FEMS Microbiol Rev. 2008;32(4):579-606.

6. Dierick K, Van Coillie E, Swiecicka I, Meyfroidt G, Devlieger H, Meulemans A, et al. Fatal family outbreak of Bacillus cereusassociated food poisoning. J Clin Microbiol. 2005;43(8):4277-9.

7. Mahler H, Pasi A, Kramer JM, Schulte P, Scoging AC, Bär W, et al. Fulminant liver failure in association with the emetic toxin of Bacillus cereus. N Engl J Med. 1997;336(16):1142-8.

8. Rau J, Perz R, Klittich G, Contzen M. [Cereulide forming presumptive Bacillus cereus strains from food--differentiating analyses using cultural methods, LC-MS/MS, PCR, and infrared spectroscopy in consideration of thermotolerant isolates]. Berl Munch Tierarztl Wochenschr. 2009;122(1-2):25-36. German. 
9. Fricker M, Messelhäusser U, Busch U, Scherer S, Ehling-

Schulz M. Diagnostic real-time PCR assays for the detection of emetic Bacillus cereus strains in foods and recent food-borne outbreaks. Appl Environ Microbiol. 2007;73(6):1892-8.

10. Ehling-Schulz M, Fricker M, Scherer S. Bacillus cereus, the causative agent of an emetic type of food-borne illness. Mol Nutr Food Res. 2004;48(7):479-87.

11. Hedberg CW, Palazzi-Churas KL, Radke VJ, Selman CA,

Tauxe RV. The use of clinical profiles in the investigation of foodborne outbreaks in restaurants: United States, 1982-1997. Epidemiol Infect. 2008;136(1):65-72.

12. Gaulin C, Viger YB, Fillion L. An outbreak of Bacillus cereus implicating a part-time banquet caterer. Can J Public Health. 2002;93(5):353-5.

13. Ankolekar C, Rahmati T, Labbé RG. Detection of toxigenic Bacillus cereus and Bacillus thuringiensis spores in U.S. rice. Int J Food Microbiol. 2009;128(3):460-6.

14. Carlin F, Fricker M, Pielaat A, Heisterkamp S, Shaheen R, Salonen MS, et al. Emetic toxin-producing strains of Bacillus cereus show distinct characteristics within the Bacillus cereus group. Int J Food Microbiol. 2006;109(1-2):132-8.

15. McIntyre L, Bernard K, Beniac D, Isaac-Renton JL, Naseby DC. Identification of Bacillus cereus group species associated with food poisoning outbreaks in British Columbia, Canada. Appl Environ Microbiol. 2008;74(23):7451-3. 